\title{
Atypically Located Pericardial Cyst in a Patient Who Underwent Off-Pump Myocardial Revascularization
}

\author{
Masahiko Ozaki, MD, PhD, ${ }^{1}$ Masanori Ogiwara, MD, PhD, ${ }^{1}$ Nobuyuki Okamura, MD, ${ }^{1}$ \\ Yu Otsu, $\mathrm{MD},{ }^{2}$ Takahiro Tanaka, $\mathrm{MD},{ }^{2}$ Yuji Kira, $\mathrm{MD}, \mathrm{PhD},{ }^{2}$ and \\ Seiichiro Shimizu, $\mathrm{MD}, \mathrm{PhD}^{3}$
}

\begin{abstract}
Pericardial cysts are rare benign mediastinal lesions and most commonly located at the cardiophrenic angle. We present a case of an atypically located pericardial cyst in a patient who underwent myocardial revascularization. A 61-year-old man with acute myocardial infarction was scheduled for coronary artery bypass grafting (CABG). Preoperative chest computed tomography revealed a homogenous cystic lesion in the superior mediastinum. The mass was located between the ascending aorta, the superior vena cava, and the left innominate vein. It was growing to the anterior of the aorta and to the right anterior paratracheal area. The density of the mass was close to that of water; thus, the contrast medium failed to enhance its visualization. A concomitant resection of the mass and the CABG was scheduled. After a medial sternotomy and bypass graft harvest, a median pericardectomy was performed. The surgeon found the cystic mass along the roof of the pericardium and located between the ascending aorta and superior vena cava. There was no adhesion between the mass and cardiovascular components. The mass was resected en bloc; therefore, off-pump CABG was completed. Histopathological examination of the resected specimen confirmed diagnosis of a pericardial cyst.
\end{abstract}

Keywords: pericardial cyst, surgery, off-pump CABG

\section{Introduction}

Pericardial cysts are rare benign mediastinal lesions. The estimated incidence of pericardial cysts is 1 per population of 100000. Pericardial cysts are most commonly

$\overline{{ }^{1} \text { Department of Cardiovascular Surgery, Showa General }}$ Hospital, Kodaira, Tokyo, Japan

${ }^{2}$ Department of Cardiology, Showa General Hospital, Kodaira, Tokyo, Japan

${ }^{3}$ Department of Pathology, Showa General Hospital, Kodaira, Tokyo, Japan

Received: August 25, 2011; Accepted: October 14, 2011 Corresponding author: Masanori Ogiwara, MD, PhD. Department of Cardiovascular Surgery, Showa General Hospital, 2-450 Tenjincho, Kodaira, Tokyo 187-8510, Japan

Email: ogiwara_showa@yahoo.co.jp

(C)2012 The Editorial Committee of Annals of Thoracic and

Cardiovascular Surgery. All rights reserved. founded in the cardiophrenic angle on routine chest roentgenograms. We present a case of atypically located pericardial cyst in a patient who underwent myocardial revascularization.

\section{Case Report}

A 61-year-old male complained of anterior chest pain, and he was immediately admitted to our hospital. The cardiologist diagnosed his illness as acute posterior myocardial infarction by laboratory examination, including an electrocardiogram. Coronary arteriography showed triple coronary vessels disease, including the left main trunk stenosis; thus, coronary artery bypass grafting (CABG) was indicated. A preoperative general survey was performed. Computed tomography revealed a homogenous cystic lesion in the superior mediastinum (Fig. 1). 


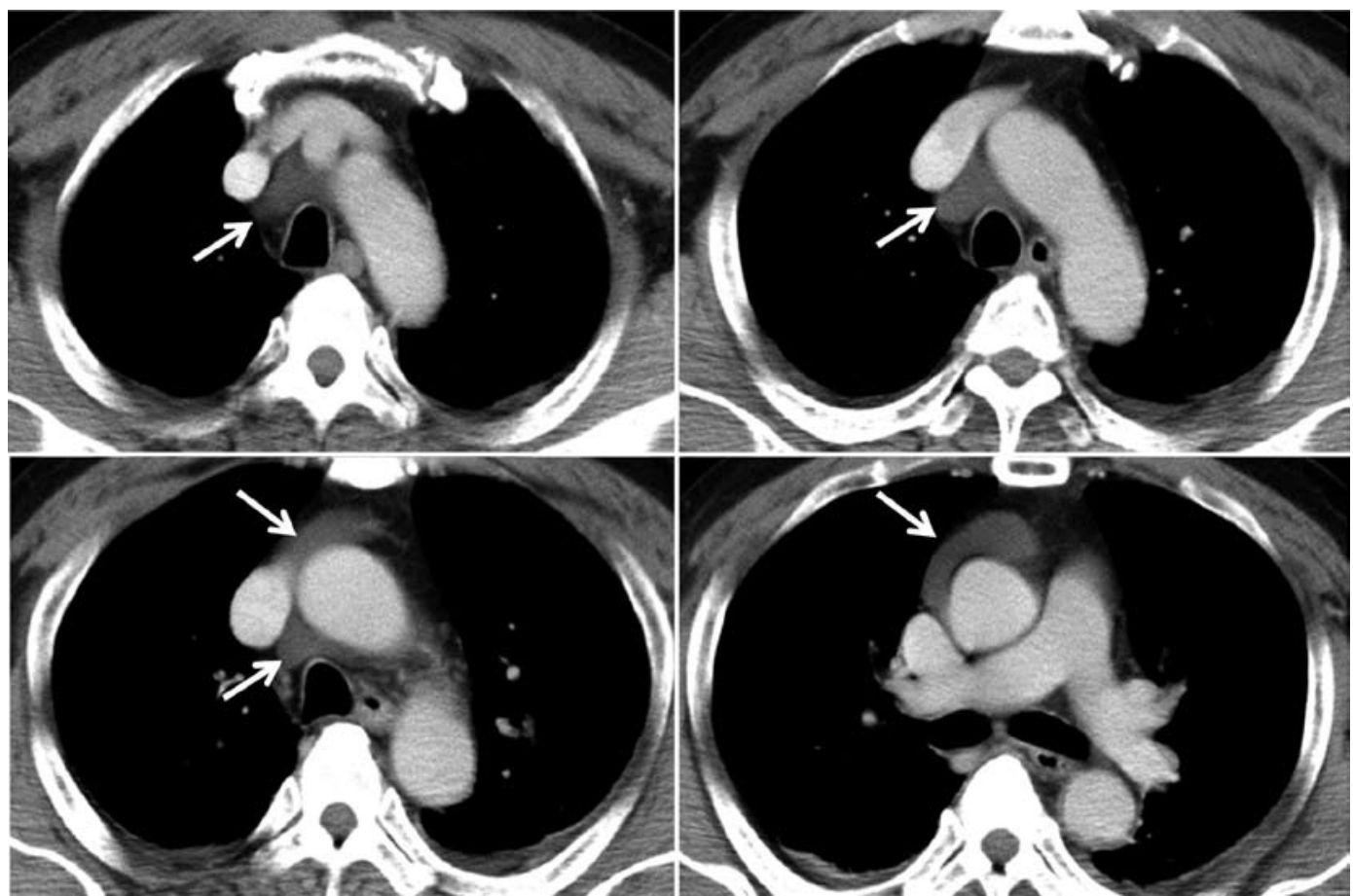

Fig. 1 Computed tomography showed a homogenous cystic lesion in the superior mediastinum (A, B, C, D, arrow heads). The mass was located between the ascending aorta, the superior vena cava, and the left innominate vein. It was growing on the anterior of the aorta and to the right anterior paratracheal area. $(\mathbf{A}, \mathbf{B}, \mathbf{C})$. The mass was thin-walled and \begin{tabular}{l|l}
$A$ & $B$ \\
\hline$C$ & $D$
\end{tabular} smooth, with a density that was similar to water. The contrast medium administration failed to enhance.

The mass was located between the ascending aorta, the superior vena cava, and the left innominate vein. It was growing towards the anterior of the aorta and to the right anterior paratracheal area. It was thin-walled and smooth with a density that was similar to water. Contrast medium administration failed to enhance the mass. A pericardial cyst was highly suspected. An operation was scheduled for concomitant resection of the mass and grafting of the coronary artery.

After inducing general anesthesia in the patient, we harvested the bypass grafts. Then, a median pericardectomy was performed. The cystic mass was communicated from the roof of the pericardium and located among the ascending aorta and superior vena cava. There was no adhesion between the mass and cardiovascular components. The inside of the mass was filled with serous, clear fluid. The mass was easily resected en bloc. Thereafter, off pump CABG was completed, and the patient was discharged uneventfully. Histopathological examination of the resected specimen showed typical mesothelial cells on the surface of the cystic lesion (Fig. 2).

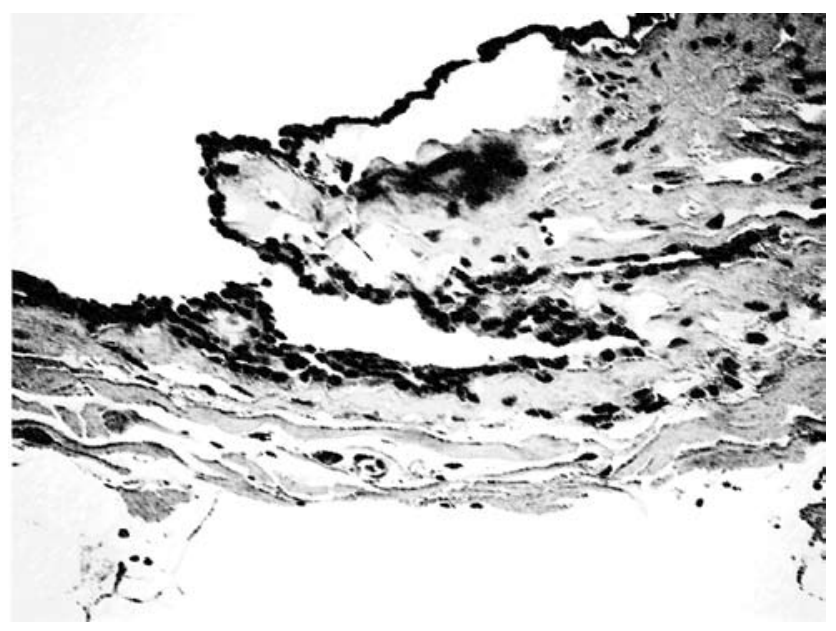

Fig. 2 Histopathological examination of the resected specimen showed typical mesothelial cells on the surface of the cystic lesion, which confirms the diagnosis of a pericardial cyst. (Hematoxylin and Eosin. $\times 200$ ) 
Those cells are positive for both calretinin and cytokeratin stains, confirming the diagnosis of a pericardial cyst (not shown).

\section{Discussion}

Pericardial cysts are uncommon, benign mediastinal lesions. The estimated incidence of pericardial cyst is 1 per population of $100000 .{ }^{1)}$

Pericardial cysts are most commonly founded at the cardiophrenic angle on routine chest roentgenograms. The right cardiophrenic angle is the more usual location (70\%), rather than the left. ${ }^{2)}$ Stroller and colleagues reported a review of e atypically located pericardial cysts, between 1929 and 1985. ${ }^{3)}$ In their review, three cases of pericardial cyst were located in the superior mediastinum, and the location of those was mentioned as rare. In 2001, Kumar and colleagues reported three cases of pericardial cyst, which were located in the superior mediastinum. ${ }^{4}$ The location and form of their cases were very similar to our case.

Computed tomography is widely accepted as an essential examination, prior to cardiovascular surgery. Especially, an evaluation of atherosclerotic degeneration of the aorta and the arch vessels, prior to coronary bypass grafting, is necessary information to obtain. Thus, cardiac surgeons will be able to perform excellent quality of bypass grafting without serious perioperative cerebrovascular events.

Pericardial cysts, usually asymptomatic, are found incidentally during a routine radiological examination. Rarely, giant pericardial cysts cause rupture, bleeding into the pericardial cavity, or compressing cardiac components and bronchus. ${ }^{3,5)}$ Cough, dyspnea, wheezing, chest pain, syncope, and arrhythmia were described in previous reports. ${ }^{3,6,7)}$

In the present case, an atypically located and asymptomatic pericardial cyst was found incidentally. We choose open surgery and resected the pericardial cyst concomitantly because coronary bypass grafting was required. According to the guideline on the pericardial diseases from the European society of cardiology, treatment for a pericardial cyst is percutaneous aspiration. ${ }^{8)}$ If this is not feasible, video assisted thoracotomy or surgical resection may be necessary. In any case, total excision of the lesion and detailed histopathological examination are necessary. ${ }^{9}$

Nevertheless, pericardial cysts do not occur in the upper mediastinum frequently. Cardiothoracic surgeons and chest physicians should include pericardial cysts in the differential diagnosis.

\section{References}

1) PERASALO O. On pericardial diverticula and their differential diagnosis. Acta Chir Scand 1953; 106: 283-91.

2) Feigin DS, Fenoglio JJ, McAllister HA, et al. Pericardial cysts. A radiologic-pathologic correlation and review. Radiology 1977; 125: 15-20.

3) Stoller JK, Shaw C, Matthay RA. Enlarging, atypically located pericardial cyst. Recent experience and literature review. Chest 1986; 89: 402-6.

4) Kutlay H, Yavuzer I, Han S, et al. Atypically located pericardial cysts. Ann Thorac Surg 2001; 72: 2137-9.

5) Shiraishi I, Yamagishi M, Kawakita A, et al. Acute cardiac tamponade caused by massive hemorrhage from pericardial cyst. Circulation 2000; 101: E196-7.

6) Thanneer L, Saric M, Perk G, et al. A giant pericardial cyst. J Am Coll Cardiol 2011; 57: 1784.

7) Neizel M, Krüger S, Spillner J, et al. A giant pericardial cyst as unusual cause for atrial flutter. J Am Coll Cardiol 2010; 55: 1160.

8) Maisch B, Seferovic PM, Ristic AD, et al. Guidelines on the diagnosis and management of pericardial diseases executive summary; The Task force on the diagnosis and management of pericardial diseases of the European society of cardiology. Eur Heart J 2004; 25: 587-610.

9) Yuncu G, Cakan A, Ors Kaya S, et al. Atypically located pericardial cysts. J Cardiovasc Surg (Torino) 2001; 42: 275-8. 